\title{
Estimação dos componentes de variância para as características de produção e de qualidade de ovos em matrizes de codorna de corte
}

\author{
Estimative of the variances components for eggs production and quality characteristics in \\ meat-type quail
}

\author{
Bruno Bastos Teixeira ${ }^{\mathrm{I}^{*}}$ Rafael Bastos Teixeira ${ }^{\mathrm{II}}$ Luciano Pinheiro da Silva \\ Robledo de Almeida Torres ${ }^{I}$ Giovani da Costa Caetano ${ }^{I}$ Ricardo Frederico Euclydes ${ }^{I}$
}

\section{RESUMO}

Dados de dois grupos genéticos UFV1 e UFV2 de matrizes de codorna de corte, com 629 animais no primeiro arquivo e 707 no segundo, foram avaliados pela técnica de componentes principais. Foram consideradas as seguintes características para análise: peso da ave (P1, P2, P3 e P4), peso médio do ovo (POM1, POM2, POM3 e POM4), peso médio da casca (PCM1, PCM2, PCM3 e PCM4), peso médio da gema (PGM1, PGM2, PGM3 e PGM4), gravidade específica média do ovo (DM1, DM2, DM3 e DM4), largura média do ovo (LOM1, LOM2, LOM3 e LOM4), comprimento médio do ovo (COM1, COM2, COM3 e COM4), número de ovos (N1, N2, N3 e N4), idade ao primeiro ovo (IDPO) e taxa de postura total (TXT). Após a coleta dos dados, foi realizada uma análise de componentes principais, buscando, com isso, selecionar as características mais explicativas. Logo as características de maior importância na variância total tiveram seus parâmetros genéticos e correlações genéticas estimados. Para o grupo genético UFV1, foram: P1, PCM1, PCM3, PGM1, PGM2, PGM4, DM4, LOM2, LOM3, COM1 e IDPO, já para UFV2: P1, P2, PCM1, PCM4, DM3, LOM2 e LOM4. As altas estimativas de herdabilidade encontradas indicam uma grande variabilidade para as características de qualidade dos ovos. As correlações genéticas mostram a possibilidade de ganhos correlacionados entre peso corporal e as características de qualidade dos ovos. Pequena resposta à seleção para produção de ovos pode ser esperada para estes grupos genéticos.

Palavras-chave: componentes principais, correlações, herdabilidade, parâmetros genéticos.

\section{ABSTRACT}

Data from two genetic groups UFV1 and UFV2 of meat-type quail, with 629 animals the first file and 707 the second, were evaluated by technique main components. The following characteristics were considered for analyses: bird weight (P1, P2, P3 and P4), egg average weight (POM1, POM2, POM3 and POM4), shell average weight (PCM1, PCM2, PCM3 and PCM4), yolk average weight (PGM1, PGM2, PGM3 and PGM4), egg average specific gravity (DM1,DM2,DM3 and DM4), egg average width (LOM1, LOM2, LOM3 and LOM4), egg average length (COM1, COM2, СOM3 and COM4), number of eggs (N1,N2,N3 and N4), and age at the first egg (IDPO). After data collecting were carried analyses of the main components out with the aim to select the most explanatory characteristics. Next the highest significant characteristics in total variance had their genetic parameters and genetic correlations estimated. For genetic group UFV1 were: P1, PCM1, PCM3, PGM1, PGM2, PGM4, DM4, LOM2, LOM3, COM1 and IDPO. For UFV2: P1, P2, PCM1, PCM4, DM3, LOM2 and LOM4. The high estimates of heritability capacity found indicate a high variability for the characteristics of egg quality. The genetic correlations indicate the possibility of correlation between body weight gains and the quality of eggs. Little response to selection for egg production can be expected for these genetic groups.

Key words: main components, correlations, heritability, genetic parameters.

\section{INTRODUÇÃO}

A coturnicultura no Brasil vem crescendo de maneira considerável e se tornando uma atividade atrativa e rentável. A razão desse sucesso é a possibilidade de rápido retorno do capital investido,

'Departamento de Zootecnia, Universidade Federal de Viçosa (UFV), 36571-000, Viçosa, MG, Brasil. E-mail: brunobatzootecnia@yahoo.com.br.*Autor para correspondência.

"Departamento de Zootecnia, Instituto Federal Minas Gerais (IFMG), Campus Bambuí, Bambuí, MG, Brasil. 
aliado à qualidade de sua carne e ao alto valor nutritivo do seu ovo.

O crescimento da coturnicultura tem encontrado barreiras que dificultam a exploração e maximização da produção. Uma dessas dificuldades seria a falta de uma avaliação econômica e zootécnica sobre as principais subespécies de codorna disponíveis para exploração, seja para produção de ovos ou carne (SILVAet al., 2007).

No Brasil, a produção de ovos é mais representativa através da subespécie Japonica, sendo que a produção de carne é proveniente do abate de machos não utilizados.

Segundo OLIVEIRA et al. (2002), com a introdução da variedade europeia, que atende os requisitos necessários à produção de carne, a exploração para corte começou a difundir-se, em virtude da taxa de crescimento elevada e do consumo de ração reduzido, constituindo uma nova alternativa para o setor avícola.

A carne de codorna é uma fonte de proteína de excelente qualidade e com grande aceitação em todas as camadas sociais. A codorna europeia é especializada para produção de carne, mas também é utilizada com o objetivo de produzir carnes e ovos.

O Brasil, no entanto, não dispõe de material genético próprio, o que deixa a coturnicultura vulnerável e dependente da importação de material genético. Algumas instituições, entretanto, começaram a desenvolver programas de melhoramento genético de codornas de corte, visando a solucionar essa dependência.

Estes programas necessitam de um acompanhamento constante das características de importância econômica de cada linhagem. A partir destes estudos é que os critérios de seleção são estabelecidos para garantir a renovação dos plantéis com animais de potencial genético superior.

Objetivou-se no presente trabalho determinar e avaliar o desempenho produtivo e reprodutivo de diferentes linhagens de matrizes de codornas de corte, estimando os parâmetros genéticos e as correlações genéticas das características selecionadas após a análise de componentes principais.

\section{MATERIAL E MÉTODOS}

Foi utilizado um banco de dados de três gerações de Coturnix coturnix, coletados de 2006 a 2008, provenientes do programa de melhoramento de aves do Departamento de Zootecnia da Universidade
Federal de Viçosa, em Viçosa, MG. Os dados foram obtidos de dois grupos genéticos de codornas de corte, sendo 629 fêmeas UFV1 e 707 fêmeas UFV2.

As características analisadas foram: peso da ave (P1, P2, P3 e P4), peso médio do ovo (POM1, POM2, POM3 e POM4), peso médio da casca (PCM1, PCM2, PCM3 e PCM4), peso médio da gema (PGM1, PGM2, PGM3 e PGM4), gravidade específica média do ovo (DM1, DM2, DM3 e DM4), largura média do ovo (LOM1, LOM2, LOM3 e LOM4), comprimento médio do ovo (COM1, COM2, COM3 e COM4), número de ovos (N1, N2, N3 e N4), taxa de postura total (TXT) e idade ao primeiro ovo (IDPO). Os números 1, 2, 3 e 4 representam os períodos de 1 a 35, 36 a 70, 71 a 105 e 106 a 140 dias de postura.

Dentro de cada período, foram avaliadas amostras de ovos de cada codorna, durante três dias consecutivos, e estas foram utilizadas para mensuração da largura e altura dos ovos e obtenção dos pesos de gema e casca.

A gravidade específica dos ovos foi obtida por meio do densímetro de óleos minerais. Para avaliar a gravidade, foram retirados todos os ovos íntegros produzidos a partir do 17음 dia de cada período e analisados em oito soluções de $\mathrm{NaCl}$, de 1,055 a 1,090 $\mathrm{g} \mathrm{cm}^{-3}$.

O peso médio dos ovos, em gramas, foi obtido pela coleta e pesagem em balança de precisão de $0,01 \mathrm{~g}$, durante três dias consecutivos a partir do $14^{\circ}$ dia do período de avaliação da produção, em quatro períodos de 35 dias. Após a pesagem, os ovos foram cortados com estilete e suas gemas separadas e pesadas. As cascas identificadas foram secas e pesadas, obtendo-se assim os pesos das cascas.

O formato do ovo altera a resistência física da casca e o índice utilizado para avaliar o seu formato é a relação largura x altura. Para sua mensuração, foi utilizado um paquímetro.

Foram realizadas análises unicaracterísticas para estimação dos componentes de variância, utilizandose o método da máxima verossimilhança restrita. O modelo estatístico utilizado na análise das características considerou o efeito fixo de Geração-eclosão e os efeitos aleatórios genético aditivo direto e residual. Esses modelos podem ser representados, na forma matricial, por $y=X \beta+Z a+e$, em que: representa o vetor de observações; a matriz de incidência dos efeitos fixos; o vetor de efeitos fixos; a matriz de incidência dos efeitos aleatórios genéticos aditivos diretos; o vetor de efeitos aleatórios genéticos aditivos diretos; e o vetor de resíduos aleatórios. As pressuposições assumidas para os efeitos aleatórios foram as seguintes: 


$$
\left[\begin{array}{l}
a \\
e
\end{array}\right] \sim N M V\left\{\left[\begin{array}{l}
0 \\
0
\end{array}\right],\left[\begin{array}{ll}
G & \phi \\
\phi & R
\end{array}\right]\right\} \text {, em que: } G=A \sigma_{a}^{2}
$$

é a matriz de (co)variâncias genéticas aditivas, sendo $A$ a matriz de numeradores do coeficiente de parentesco e $\sigma_{a}^{2}$ a variância genética aditiva; $R=I \sigma_{e}^{2}$ a matriz de variância residual, sendo $I$ a matriz identidade e $\sigma_{e}^{2}$ a variância residual.

Também foram realizadas análises bicaracterísticas para estimação das covariâncias e correlações genéticas. As estimativas dos componentes de (co)variância e dos parâmetros genéticos foram obtidas pelo programa MTDFREML (Multiple Trait Derivative-Free Restricted Maximum Likelihood), descrito por BOLDMAN et al. (1995), que utilizaram a metodologia da máxima verossimilhança restrita livre de derivadas (DFREML). O MTDFREML utiliza o algoritmo simplex para localizar o mínimo de - $2 \log _{e} \mathrm{~L}$ (L = função de verossimilhança), e os componentes de (co)variância que minimizam a função $-2 \log _{e} L$ são estimativas de máxima verossimilhança.

Como critério de convergência, utilizou-se a variância dos valores do simplex (-2 $\log _{\mathrm{e}}$ de verossimilhança), menores do que $10^{-9}$. Após cada convergência, o programa foi reiniciado, usando as estimativas obtidas anteriormente, como valores iniciais. Esse procedimento foi repetido até que as diferenças entre as estimativas das duas últimas convergências fossem menores que $10^{-4}$.

\section{RESULTADOS E DISCUSSÃO}

\section{Grupo Genético UFV1}

As características utilizadas após análise de componentes principais foram: peso da ave no primeiro período (P1), peso médio da casca no primeiro e terceiro período (PCM1 e PCM3), peso médio da gema no primeiro, segundo e quarto período (PGM1, PGM2 e PGM4), gravidade específica média do ovo no quarto período (DM4), largura média do ovo no segundo e terceiro período (LOM2 e LOM3), comprimento médio do ovo no primeiro período (COM1) e idade ao primeiro ovo (IDPO). A produção de ovos foi também acrescentada para análise da característica taxa de postura total (TXT).

As estimativas de herdabilidade e correlações genéticas para as características estudadas são apresentadas na tabela 1 . As estimativas de herdabilidade para características de qualidade dos ovos (PCM1, PCM3, DM4, PGM1, PGM2, PGM4,LOM2, LOM3 e COM1) apresentaram valores elevados no grupo genético UFV1. Isso se deve ao fato de esses animais terem sua seleção focada apenas na característica peso corporal e, com isso, apresentam ainda alta variabilidade genética, em relação a aspectos da qualidade dos ovos.

Isso indica a possibilidade de estudos buscando uniformizar esses ovos, por meio da seleção de matrizes que atendam ao padrão de ovo estabelecido. Essa uniformização seria importante no aspecto econômico, pois, no caso de ovos pequenos demais, a legislação brasileira (Decreto n.56.585 de 20 de julho de 1965) exige um mínimo de peso por dúzia. Por outro lado, no caso de ovos grandes demais, haveria maior pressão nas bandejas inferiores, durante a coleta, com consequente maior índice de quebra. Essa uniformidade pode ser um padrão exigido pelo consumidor futuramente.

Os valores de herdabilidade encontrados para peso de gema (PGM1, PGM2, PGM4) e peso da casca (PCM1 e PCM3) mostram outro aspecto importante que não vem recebendo atenção por parte dos produtores e pesquisadores, que seria em relação à eclodibilidade dos ovos. A grande variabilidade existente para essas características pode ser a responsável pela variação no tempo de incubação e nascimento das codorninhas (entre 17 e 21 dias).

O atraso ou a extensão nesse tempo de incubação pode reduzir a eclodibilidade, quando o manejo estabelece a retirada num tempo pré-fixado. A extensão do período, com vistas a otimizar a qualidade de codornas no nascimento ou alojamento, ganhou maior atenção com a determinação dos efeitos negativos nos parâmetros de performance pósnascimento, decorrente do atraso no fornecimento de ração e(ou) água.

Considerando que o peso ao nascimento está correlacionado com os componentes dos ovos, a uniformização dos ovos poderia ser praticada para homogeneizar o nascimento, o que viria a reduzir o estresse das aves nessa fase inicial, gerando melhor desempenho das codornas.

As características da qualidade dos ovos tiveram valores de correlações genéticas positivas e altas entre si, mostrando que a seleção de apenas uma característica levará a ganhos correlacionados nas demais características. Esse resultado sugere que algumas mensurações que são trabalhosas e dependentes do avaliador não precisariam ser medidas.

As características de qualidade dos ovos correlacionam-se genética e negativamente com a taxa de postura total e, sendo assim, as melhorias que poderiam ser obtidas pela seleção dessas características provocariam uma redução na produção de ovos. 
Tabela 1 - Estimativas das herdabilidades (diagonal e sublinhado) das análises unicaracterísticas, correlações genéticas aditivas (acima da diagonal) das análises bicaracterísticas do grupo UFV1.

\begin{tabular}{|c|c|c|c|c|c|c|c|c|c|c|c|c|}
\hline$h^{2}$ & LOM2 & LOM3 & COM1 & PGM1 & PGM2 & PGM4 & PCM1 & PCM3 & DM4 & $\mathrm{P} 1$ & IDPO & TXT \\
\hline LOM2 & $\underline{0,38}$ & 0,97 & 0,79 & $*$ & 0,68 & 0,78 & 0,72 & 0,65 & $-0,11$ & 0,62 & 0,14 & $-0,68$ \\
\hline LOM3 & & $\underline{0,49}$ & 0,69 & $*$ & 0,55 & 0,72 & 0,63 & 0,66 & $-0,08$ & 0,46 & 0,38 & $-0,44$ \\
\hline COM1 & & & $\underline{0,24}$ & * & 0,6 & 0,83 & 0,72 & 0,58 & 0,03 & 0,57 & 0,29 & $-0,21$ \\
\hline PGM1 & & & & $\underline{0,24}$ & $*$ & $*$ & $*$ & $*$ & $*$ & $*$ & $*$ & $*$ \\
\hline PGM2 & & & & & $\underline{0,33}$ & 0,96 & 0,64 & 0,5 & $-0,11$ & 0,62 & 0,37 & $-0,41$ \\
\hline PGM4 & & & & & & $\underline{0,32}$ & 0,01 & 0,74 & 0,25 & 0,19 & 0,18 & $-0,36$ \\
\hline PCM1 & & & & & & & $\underline{0,54}$ & 0,95 & 0,69 & 0,4 & $-0,16$ & $-0,12$ \\
\hline РСM3 & & & & & & & & $\underline{0,49}$ & 0,8 & 0,22 & 0,16 & $-0,23$ \\
\hline DM4 & & & & & & & & & $\underline{0,44}$ & $-0,05$ & $-0,61$ & $-0,22$ \\
\hline $\mathrm{P} 1$ & & & & & & & & & & $\underline{0,34}$ & $-0,01$ & $-0,14$ \\
\hline IDPO & & & & & & & & & & & $\underline{0,10}$ & $-0,85$ \\
\hline TXT & & & & & & & & & & & & $\underline{0,09}$ \\
\hline
\end{tabular}

*Não convergiu pelo critério adotado de variância dos valores do simplex (-2loge de verossimilhança) menores do que $10^{-9}$; LOM2 LOM3: largura média do ovo; COM1: comprimento médio do ovo; PGM1 PGM2 PGM4: peso médio de gema; PCM1 PCM3: peso médio da casca; DM4: gravidade específica média do ovo; P1: peso da ave; IDPO: idade ao primeiro ovo; TXT: taxa de postura total.

A herdabilidade estimada de P1 é 0,34, o que indica uma possibilidade de resposta à seleção massal. A característica P1 correlaciona-se genética e positivamente com as características qualitativas dos ovos, o que é importante para produção de codorninhas de qualidade. A produção de ovos total apresentou correlação genética negativa $(-0,14)$ com P1. MINVIELLE (1998) descreveu valores entre correlação genética do número de ovos e peso corporal que variaram de 0 a - 0,21 , o que indica margem razoável de ganhos genéticos no número de ovos sem grandes alterações no peso corporal. O grupo genético UFV1 pode ser selecionado para aumentar o peso corporal, sem que haja grandes perdas no número de ovos por matriz alojada.

Uma estimativa de herdabilidade de 0,09 foi obtida para a característica idade ao primeiro ovo, fato que se deve à grande influência do meio ambiente nesta característica, caracterizado por fatores como época de nascimento, nutrição, etc. Essas matrizes apresentam menor variabilidade, pois foram selecionadas aos 28 dias de idade, quando são alojadas em gaiolas, tornando-as mais homogêneas.

Uma estimativa de herdabilidade de 0,09 foi obtida também para a taxa de postura total, o que indica a pouca possibilidade de alterar a produção por meio de seleção. SILVA et. al. (2007), ao estudarem codornas de postura, encontraram estimativas de herdabilidade praticamente nulas para produção de ovos até os 90 dias.

\section{Grupo Genético UFV2}

As características utilizadas após análise de componentes principais foram: peso da ave no primeiro e segundo período (P1 e P2), peso médio da casca no primeiro e quarto período (PCM1 e PCM4), gravidade específica média do ovo no terceiro período (DM3), largura média do ovo no segundo e quarto período (LOM2 e LOM4), comprimento médio do ovo no terceiro período (COM3), idade ao primeiro Ovo (IDPO) e número de ovos no segundo período (N2). A produção total de ovos foi também analisada, através da inclusão da característica taxa de postura total (TXT).

As estimativas de herdabilidade e correlações genéticas para as características estudadas são apresentadas na tabela 2. Nota-se que o grupo genético UFV2 apresentou resultados semelhantes em relação às características da qualidade dos ovos obtidos pelo grupo genético UFV1. Isso se deve ao fato de os dois grupos genéticos terem sido submetidos às mesmas práticas de manejo e método de seleção (pertencem ao mesmo programa de melhoramento genético).

Menores estimativas de herdabilidade para as características P1 e P2 foram obtidas para o grupo genético UFV2, o que mostra menor variabilidade das codornas para essas características, consequência da maior intensidade de seleção praticada nestas aves anteriormente ao estabelecimento do programa.

Observou-se correlação genética negativa entre P1 e as características N2 e TXT, o que pode ocasionar redução no número de ovos e consequentemente na produção total de ovos, com o aumento do peso das aves, característica esta utilizada até o presente como único critério de seleção do programa.

O grupo genético UFV2 apresentou estimativas de herdabilidade para N2 de 0,03 e TXT de 
Tabela 2 - Estimativas das herdabilidades das análises unicaracterísticas (diagonal e sublinhado), e correlações genéticas aditivas (acima da diagonal) das análises bicaracterísticas do grupo UFV2.

\begin{tabular}{|c|c|c|c|c|c|c|c|c|c|c|c|}
\hline$h^{2}$ & COM3 & DM3 & IDPO & LOM2 & LOM4 & P1 & $\mathrm{P} 2$ & PCM1 & PCM4 & N2 & TXT \\
\hline COM3 & $\underline{0,27}$ & 0,16 & $-0,29$ & 0,6 & 0,48 & 0,32 & 0,45 & 0,74 & 0,65 & 0,63 & 0,45 \\
\hline DM3 & & $\underline{0,51}$ & 0,33 & $-0,32$ & $-0,19$ & $-0,27$ & 0,23 & 0,41 & 0,54 & 0,17 & $-0,14$ \\
\hline IDPO & & & $\underline{0,09}$ & 0,09 & 0,24 & 0,18 & 0,45 & $-0,13$ & 0,49 & $-0,95$ & $-0,03$ \\
\hline LOM2 & & & & $\underline{0,43}$ & 0,96 & 0,71 & 0,56 & 0,66 & 0,35 & $-0,1$ & 0,02 \\
\hline LOM4 & & & & & $\underline{0,49}$ & 0,57 & 0,4 & 0,57 & 0,59 & 0,14 & 0,22 \\
\hline P1 & & & & & & $\underline{0,21}$ & 0,84 & 0,25 & 0,2 & $-0,44$ & $-0,17$ \\
\hline P2 & & & & & & & $\underline{0,22}$ & 0,61 & 0,6 & 0,24 & 0,24 \\
\hline PCM1 & & & & & & & & $\underline{0,27}$ & 0,91 & $-0,15$ & $-0,23$ \\
\hline PCM4 & & & & & & & & & $\underline{0,57}$ & $-0,09$ & $-0,09$ \\
\hline N2 & & & & & & & & & & $\underline{0,03}$ & 0,89 \\
\hline TXT & & & & & & & & & & & $\underline{0,07}$ \\
\hline
\end{tabular}

COM3: comprimento médio do ovo; DM3: gravidade específica média do ovo; IDPO: idade ao primeiro ovo; LOM2 LOM4: largura média do ovo; P1 P2: peso da ave; PCM1 PCM4: peso médio da casca; N2: número de ovos; TXT: taxa de postura total.

0,07. Esses valores sugerem uma pequena resposta à seleção para número de ovos ou taxa de postura para este grupo genético.

Os valores de coeficientes de herdabilidade para peso de casca encontrados para ambas as linhagens demonstram a possibilidade de se poder melhorar a qualidade de casca, diminuir a perda por transporte e promover maior homogeneidade ao nascimento e na eclodibilidade.

\section{CONCLUSÃO}

A variabilidade encontrada nas características de qualidade dos ovos, nas matrizes selecionadas para produção de codorna de corte do programa de melhoramento de codorna da UFV sugerem a possibilidade de ganhos correlacionados nessas características pela seleção massal do peso corporal. Para produção de ovos, pode ser esperada uma pequena resposta à opção para seleção massal desses grupos genéticos.

\section{REFERÊNCIAS}

BOLDMAN, K.G.A et al. A manual for use of MTDFREML. A set of programs to obtain estimates of variance and covariances. Washington, DC: ARS, USDA, 1995. 120p.

SILVA, R.M. et. al. Parâmetros genéticos para peso do ovo e produção de ovos em três linhagens de codorna de postura. In: SIMPÓSIO INTERNACIONAL DE COTURNICULTURA, 2.; CONGRESSO BRASILEIRO DE COTURNICULTURA, 3., 2007, Lavras, MG. Anais... Lavras: UFLA, 2007. p.213.

MINVIELLE, F. Genetics and breeding of Japanese quail for production around the world. In: ASIAN PACIFIC POULTRY CONGRESS, 6., 1998, Nagoia. Proceedings... Nagoia: Japan Poultry Science Association, 1998. p.122-127.

OLIVEIRA, N.T.E. et al. Exigências de proteína bruta e energia metabolizável para codornas japonesas machos criadas para a produção de carne. Arquivo Brasileiro de Medicina Veterinária e Zootecnia, v.54, p.196-203, 2002. Disponível em: <http://www.scielo.br/pdf/rbz/v31n2/10354>. Acesso em: 24 set. 2008. 